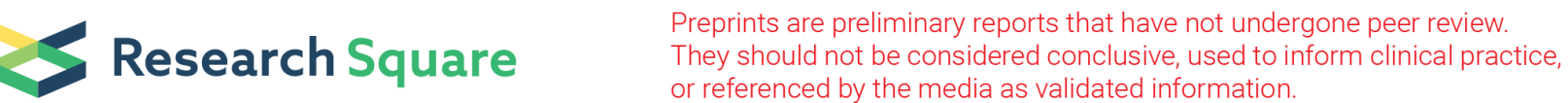

\section{Targeted Delivery of Intranasally Administered Cyclovirobuxine D Liposomes to Brain for Treatment Cerebral Ischemia-Reperfusion Injury via Anti- oxidative Stress and Activating Autophagy}

\section{Tuo Liu}

Institute of Chinese Materia Medica, China Academy of Chinese Medical Sciences

Fang Yang

School of Pharmaceutical Sciences, Guangzhou University of Chinese Medicine

Xiangyi Lu

School of Pharmaceutical Sciences, Guangzhou University of Chinese Medicine

Chang Liu

School of Pharmaceutical Sciences, Guangzhou University of Chinese Medicine

\section{Yang Yu}

School of Pharmaceutical Sciences, Guangzhou University of Chinese Medicine

\section{Zhilian Chen}

First Affiliated Hospital of Guangzhou University of Chinese Medicine

Yongling Long ( $\square$ longyongling@gzucm.edu.cn)

School of Pharmaceutical Sciences, Guangzhou University of Chinese Medicine

\section{Research Article}

Keywords: Cyclovirobuxine D, Cerebral Ischemia-Reperfusion Injury, Nose to brain delivery, Brain targeting liposomes, Oxidative stress, Autophagy

Posted Date: January 3rd, 2022

DOI: https://doi.org/10.21203/rs.3.rs-966870/v2

License: (c) (i) This work is licensed under a Creative Commons Attribution 4.0 International License.

Read Full License 


\section{Abstract}

The lack of effective therapy mandates development of treatment for cerebral ischemia-reperfusion injury (CIRI. The previous study suggested that Cyclovirobuxine D (CVBD) encapsulated in Angiopep-conjugated Polysorbate 80-Coated Liposomes showed a better brain targeting by intranasal administration. Therefore, this study focused on the protection and mechanism of CVBD brain-targeted liposomes in treating CIRI. In order to evaluate these, the CIRI rat model was induced by middle cerebral artery occlusion (MCAO)-reperfusion. Pharmacological evaluation was assessed in vivo by general indexs, neurobehavioral scores, triphenyl tetrazolium chloride (TTC) staining, histopathological staining (HE staining and Nissl staining), small animal magnetic resonance imaging, biochemical assay and Western blot. The results show that CVBD liposomes alleviated pathological damage of brain. Futhermore, the protective effect of CVBD liposomes on OGD/R-injured HT22 cell was investigated by cell fusion degree, cell proliferation curve and cell viability. OGD/R-injured HT22 cell was infected by mRFP-GFP-LC3 adenovirus. The autophagosome and autophagy flow were observed by laser confocal microscopy, and autophagy-related protein expressions (LC3, p62 and Beclin 1) were analyzed by Western blot. Meanwhile, the classic autophagy inhibitor, chloroquine, was used to explore the autophagy-regulated mechanism of CVBD brain-targeted liposomes in treating CIRI. In cell model of oxygen and glucose deprivation/reoxygenation, CVBD liposomes increased cell viability and decreased ROS level. CVBD liposomes improved oxidative stress protein expressions and activated autophagy in vitro. Furthermore, CVBD liposomes reversed the decrease of cell viability, increase of ROS level, and reduction of protein expressions associated to anti-oxidative stress and autophagy induced by chloroquine. Collectively, CVBD liposomes inhibited CIRI via regulating oxidative stress and enhancing autophagy level in vivo and in vitro, showing a great potential in treating CIRI in clinic.

\section{Graphical Abstract}

In this study, tween-80-coated CVBD brain-targeted liposomes were prepared by thin-film dispersion method, and the brain targeting efficiency was improved by Angiopep-2 modification and intranasal administration. The results showed that it could reduce the pathological damage of the brain and make the blood flow partly back to normal for the rats in the cerebral ischemia reperfusion injury model. Furthermore, CVBD brain-targeted liposomes increased cell viability, reduced ROS injury, improved oxidative stress protein expressions, activated autophagy and autophagy flux in vitro (Figure Graphical Abstract).

\section{Introduction}

Ischemia-reperfusion injury is caused by a blood reperfusion injury in ischemic brain tissue, and usually occurs in the treatment stage of ischemic stroke, which can aggravate brain tissue injury [1-3]. Therefore, new strategies focusing on CIRI are urgently needed. 
Cyclovirobuxine D (CVBD) is a steroidal alkaloid extracted from the Chinese medicinal herb Buxux microphylla, which is widely used in the form of Huang yangning tablets to treat cardiovascular and cerebrovascular diseases by oral [4]. JLX001 is a novel compound of the dihydrochlorid of cyclovirobuxine $\mathrm{D}$ (CVBD) \the protective effect on cerebral ischemia-reperfusion injury focal cerebral ischemia has been proved $[5,6]$. Nevertheless, research on CVBD improving cerebral ischemia-reperfusion injury is rare. These applications may be attributed to the effect of CVBD on symptoms of hypoxia [7]. However, the existence of blood-brain barrier (BBB), which hinders the passage of molecules from the blood into the cerebral compartment, limits the applications of CVBD on brain disorders.

Targeted delivery of intranasally administered nano delivery system preparation has been widely accepted as a practical non-invasive approach, which can bypass the BBB and rapidly deliver agents including macromolecules and small molecules to the brain, thereby reducing systemic exposure of therapeutics and potential systemic adverse effects [8-10]. Liposomes in nano delivery system has great potential for organ targeting, biocompatibility and inclusiveness and has resulted in wide public attention in the recent years [11-13]. In addition, the surface of the liposome can undertake structural modifications, such as targeting by transferrin, lactoferrin insulin and low-density lipoprotein receptors to realize drug delivery to the brain. Angiopep-2, a kind of new polypeptide, can pass to brain by endocytosis mediated by low density lipoprotein receptor, and its penetration efficient through BBB is dozens of times more than transferrin [14-16].

In the previous study, we prepared cyclovirobuxine D encapsulated in Angiopep-2 conjugated Polysorbate 80-Coated Liposomes (T80-An2-CVB-D-Lps). The T80-An2-CVBD-Lps with an entrapment efficiency (EE) of $92.39 \pm 1.29 \%$ had a narrow polydispersity index $(\mathrm{PI})(0.301 \pm 0.01)$ and were $72.03 \pm 0.33 \mathrm{~nm}$ in size with a zeta potential of $-15.23 \pm 0.61 \mathrm{mV}$. The prepared liposomes had small particle size, uniform distribution and stable quality, and no hemolysis was observed. Besides, there was a better targeting and higher AUC of CVBD in the brain after T80-An2-CVBD-Lps by intranasal administration [17-19].

Based on the superiority of nasal administration and nano preparations, therefore, this study focused on the protection of CVBD brain-targeted liposomes in CIRI to clarify the target and molecular mechanism of CVBD in treating CIRI, which can lay a foundation for innovative research and development of stroke.

\section{Materials And Methods}

\section{Materials and Reagents}

Cyclovirobuxine D (CVBD) (>98\% purity) was purchased from Sichuan Weikeqi Biological Technology Co., Ltd. (Chengdu, China). Soybean lecithin (injection grade, $>98 \%$ purity), cholesterol, polysorbate 80 and DLa-Tocopherol were purchased from Shanghai Aladd in Biochem Technology Co., Ltd. (Shanghai, China). Angiopep-2 (TFFYGGSRGKRNNFKTEEY) was synthesized by GL Biochem Co., Ltd. (Shanghai, China). DSPE-PEG-2000 was purchased from Xi'an ruixi Biological Technology Co., Ltd. (Xian, China). Nimodipine 
(NMDP) was purchased from Bayer Healthcare Co., Ltd. (Beijing, China). Dulbecco's modified Eagle's medium (DMEM), fetal bovine serum (FBS), and $0.25 \%(\mathrm{w} / \mathrm{v})$ trypsin solution were purchased from Gibco (Grand Island, New York, USA). All other chemicals were of analytical or chromatographic grade.

\section{Experimental Animals}

Eighty male Sprague-Dawley rats of SPF grade (250-270 g, approval No. SYXK 2018-0001) were provided by the Experimental Animal Center of Guangzhou University of Chinese Medicine. All rats had free access to a standard diet and drank water, and they were housed in a room at $24.0 \pm 0.5^{\circ} \mathrm{C}$ with a $12 \mathrm{~h} / 12 \mathrm{~h}$ cyclic lighting schedule. This study was approved by the Animal Ethics Committee of Guangzhou University of Chinese Medicine (Approved No. S2018034).

\section{Cerebral Artery Occlusion (MCAO)-Induced Focal CIRI Model}

MCAO-induced CIRI was established as follows [20]. Rats were anesthetized via an intraperitoneal injection of pentobarbital sodium. Then, the left common carotid artery (CCA), internal carotid artery (ICA), and external carotid artery (ECA) were carefully exposed. The ECA needed to be ligated at a proximal location. The CCA and ICA were temporarily nipped. A suture was inserted from an incision on the CCA into the ICA until the head of the suture arrived at the bifurcation of the ICA and middle cerebral artery (MCA). The length of the nylon suture was approximately $18 \mathrm{~mm}$ from the bifurcation of the CCA/ECA. After $1.5 \mathrm{~h}$, the suture was removed to restore blood supply to the MCA area via reperfusion. After the surgery, neurological deficits were evaluated and scored. The rats in the Sham group underwent the same surgical procedure without a suture occlusion.

\section{Study Design}

All animals were randomly and averagely divided into four groups $(n=20)$ as follows: Sham (distilled water, p.o.), Model (distilled water, p.o.), NMDP (NMDP at a dosage of $0.03 \mathrm{~g} / \mathrm{kg}$, p.o.), and The T80-An2CVBD-Lps group (CVBD) (CVBD at the dosage of $0.2 \mathrm{~mL} /$ per rat, Nasal drip). Distilled water, NMDP or CVBD were administered once daily for a week after surgery.

\section{Neurological Behavior Assessment}

Neurologic deficit scoring was performed on days 1, 3 and 7 after surgery. The criteria were as follows: (1) lifting rats by the tail and observing the flexion-extension of their front legs (0-4 points); (2) placing rats on the ground and examining the resistance force of their shoulders (0-3 points); (3) placing rats on a 
metal net and examining the muscular tension of their front legs (0-3 points); and (4) circling to the left or right (0-3 points). Higher scores indicated more severe neurological deficits.

\section{Cerebral Infarction Size}

Rats were sacrificed using anesthesia, and then whole heads were removed via decapitation for sample collection. Cerebrums were completely peeled off, and other tissues were carefully removed, including cerebella, olfactory bulbs, and low brain stems. The sample-collecting process was performed on ice. Samples were quickly frozen at $-20^{\circ} \mathrm{C}$ for $30 \mathrm{~min}$ and later removed to be made into coronal sections. These sections were further preprocessed as follows: stained in $2 \%$ TTC (2,3,5-triphenyl-2H-tetrazolium chloride, Amresco, center valley, PA, USA), incubated in the dark for 20 min, and then fixed in $4 \%$ paraformaldehyde (Servicebio, Wuhan, Hubei, China) for $1 \mathrm{~h}$. The TTC staining results were digitally scanned and analyzed using Image-Pro Plus 6.0.

\section{Pathological Staining}

Six rats from each group were used for pathological analysis. The cerebral tissue around the optic chiasma were sliced into coronal sections, fixed in $4 \%$ paraformaldehyde, dehydrated through a graded alcohol series, mounted in paraffin, cut into cross-sections at $4 \mu \mathrm{m}$, and stained with hematoxylin \& eosin (HE staining) or $0.1 \%$ cresyl violet-luxol (Nissl staining). HE staining was used to evaluate the pathological changes of cerebral tissue, and Nissl staining was used to evaluate the morphology, amount, and distribution changes of Nissl bodies.

\section{MRI Scanning}

The MRI study of the characteristics of the ischemic injury of rat brains and blood vessels was carried out using small animal 7T PharmaScan70/16 US (Bruker BioSpin, USA). One animal from each group was randomly selected for the MRI scan on the 7th day after the MCAO operation. The rats were anesthetized with $5 \%$ isoflurane-nitrogen/oxygen $(70 \% / 30 \%)$ mixed gas and maintained with $1 \%$ isoflurane. The specific parameters of T2 WI imaging are as follows: $T E=30.5 \mathrm{~ms}$, TR=3000 ms, layer thickness $=0.8 \mathrm{~mm}$. The parameters of 3D-TOF MRA imaging are set as follows: TE $=2.7 \mathrm{~ms}, \mathrm{TR}=15 \mathrm{~ms}$, layer thickness $=0.3$ $\mathrm{mm}$. After scanning, the raw data obtained by ParaVision 6.0.1, and analysed by RadiAnt DICOM Viewer.

\section{Biochemical Indices}

The impact of CVBD on oxidative stress in acute ischemic stroke models was assessed by lipid peroxidation (LPO), superoxide dismutase (SOD), malondialdehyde (MDA) and catalase (CAT). The measurements and calculations were strictly detected with commercial kits (Jian-cheng, Nanjing, Jiangsu, China). 


\section{Western Blot}

Western blot was applied to detect protein expressions of LC-3, Nrf2, HO-1, NQ01, GCLM, p62 and Beclin1 (Abcam, MA, USA). Briefly, tissues were lysed in RIPA buffer (Cwbio, Beijing, China) and centrifuged at $12,000 \times \mathrm{g}, 4^{\circ} \mathrm{C}$ for $10 \mathrm{~min}$. After dilution, degeneration and centrifugation, the supernatant was added to a prepared sodium dodecyl sulfate-polyacrylamide gel to separate proteins via electrophoresis. Proteins were then transferred to a polyvinylidene difluoride membrane. After blocking with $5 \%$ bovine serum albumin for $1 \mathrm{~h}$, the membrane was probed overnight at $4^{\circ} \mathrm{C}$ with anti-NQ01, anti-LC3B, anti-GCLM, antiactive p62, anti-Beclin1, anti-Nrf2, anti-heme oxygenase 1 (Abcam, MA, USA), anti-GAPDH or anti- $\beta$-actin antibodies (Cwbio, Beijing, China). The membrane was incubated with secondary antibody (IRDye 800CW Goat anti-Rabbit or IRDye $800 \mathrm{CW}$ Goat anti-Mouse were purchased from LI-COR, Inc (Lindon, Nebraska, USA)). Bands were quantified using Image-Pro Plus 6.0.

\section{Cell Culture}

HT22 cells were kindly gifted by Prof. Wang Lisheng from Guangzhou University of Chinese Medicine. Cells were cultured in DMEM supplemented with $10 \%$ fetal bovine serum $(\mathrm{v} / \mathrm{v})$ and antibiotics (100 units/l penicillin, $100 \mathrm{mg} / \mathrm{l}$ streptomycin) at $37^{\circ} \mathrm{C}$ in an incubator with $5 \% \mathrm{CO}_{2}$.

\section{Establishment of OGD/R Cell Model and Cell Treatment}

After the culture medium was replaced with glucose-free DMEM at $37^{\circ} \mathrm{C}$ in an incubator with $5 \% \mathrm{O}_{2}$ for 9 $\mathrm{h}$, the cells were placed with complete medium and then incubated with $20 \% \mathrm{O}_{2}$ for $1 \mathrm{~h}$ to finish the process of $\mathrm{OGD} / \mathrm{R}$. Cells were divided into the following groups: Normal group (NC) (cells were cultured in normal environment without any stimuli); OGD/R group; OGD/R + $9.38 \mu \mathrm{M}$ CVBD liposomes (CVBD-L);

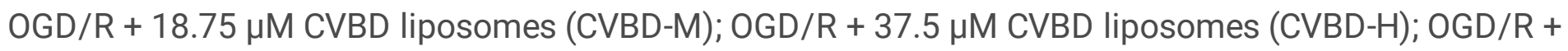
chloroquine (CQ); OGD/R + CQ + 37.5 $\mu \mathrm{M}$ CVBD liposomes. CQ (S4157, Selleck, TX, USA) was the specific inhibitor of autophagy and the applied concentration in the study was $25 \mu \mathrm{M}$. Cell viability was measured by CCK-8 kits. ROS was detected by commercial kits (E004, Jian-cheng, Nanjing, China) and analyzed by Real-Time Live-Cell Imaging System (IncuCyte ZOOM, Essen Bioscience, USA).

\section{Mrfp-GFP-LC3 Adenovirus Transfection}

After transfected, cells were treated with/without OGD/R according to the mentioned above. mRFP-GFPLC3 adenoviral vectors were purchased from HanBio Technology (Shanghai, China). Adenoviral infection was performed according to the manufacturer's instructions. LC3 puncta were examined with Zeiss LSM710 confocal microscope (Carl Zeiss) fitted with a 40x oil immersion objective [21] . 


\section{Statistical Analysis}

Data were analyzed using SPSS 22.0 statistical software and presented as the mean \pm SD. A $t$-test was used to compare two independent samples, whereas one-way analysis of variance was applied to analyze between-group differences. In addition, a least significant difference test was used for twosample comparisons between groups. $P<0.05$ was considered statistically significant. Graphs were created using GraphPad Prism 6.0.

\section{Results}

Establishing of Rat MCAO Model and Pharmacodynamics Evaluation of CVBD Brain-targeted Liposomes in vivo

Compared with Sham group, the neurobehavioral scores in Model group, CVBD group and NMDP group were significantly increased by $580 \%$ at day 1 . The scores in CVBD group and NMDP group were decreased over time. On the seventh day, the neurobehavioral scores in CVBD group and NMDP group were significantly decreased by about $50 \%$ as compared with Model group (Figure 1A).

TTC staining showed that no ischemic infarct lesion was found in the brain tissue of Sham group. Compared with Sham group, there was obvious ischemic infarct lesion, and the ischemic area in Model group was significantly increased by about 20 times. Compared with Model group, the cerebral ischemic area of CVBD group and NMDP group decreased significantly by about 60\% (Figure 1B).

HE staining showed that no obvious cerebral ischemia was found in Sham group. Ischemic necrosis in the left cerebral cortex was found in Model group. There were large areas of softening lesion, liquefactive necrosis of neural tissue, disappearance of dissolution of neuronal nuclei, and a lot of microglia infiltration. Some nuclei of pyramidal cells in the hippocampus were hyperchromatic and some were even hollow. Compared with Model group, the pathological damage of brain tissue in CVBD group and NMDP group was significantly allevited, and to be specific, the neuronal cell bodies in the cortex were shrunk and triangle-shaped, the nuclei were hyperchromatic, the nucleoli and chromatin could not be distinguished, and no obvious abnormality was found in the hippocampus (Figure 1C).

Nissl Staining showed that 4-5 layers of blue-stained neurons can be seen through the Nissl staining, the Nissl bodies were abundant, and the cells were well-defined in the Sham group. In the Model group, the nerve cell layers became thinner and less dense, and the number of Nissl bodies was decreased greatly. Compared with Model group, pathological changes of brain tissue in CVBD group and NMDP group were significantly reduced, and there were more surviving cells and more orderly-arranged cell layers, only a few pyramidal cells were decreased (Figure 1D).

\section{Imaging Evaluation of CVBD Brain-targeted Liposomes for the Treatment of CIRI}


On the 7th day after operation, MRI-T2WI weighted imaging technique was used to observe the brain injury of rats in vivo. The MRI-T2 weighted images of the head in Sham group showed no infarct lesion, and the cortex, striatum and hippocampus were symmetrical. In Model group, there was a large area of bright sport in the right side of the brain and MR signal enhancement which was caused by MCAO. In CVBD group and NMDP group, the bright spot still existed, but the signal area of abnormal tissue was decreased and the volume of normal tissue was increased significantly (Figure 2A).

In addition, MRA-3D TOF angiography was used to observe the repair of cerebral vascular injury in rats in vivo. The results showed that no infarct lesions, normal blood flow and clear blood vessels were found in the blood vessels of Sham group. In Model group, there was a large area with no signal in the right cerebral vessels and reduced blood flow which caused by MACO. In CVBD group and NMDP group, the area with no signals in the right cerebral vessels still existed, but the area with signal decreased significantly, and blood flow was partly normal and blood vessels were partly clear (Figure 2B).

\section{Effects of CVBD Brain-targeted Liposomes on Oxidative Stress and Autophagy in MCAO Rats}

The results showed significantly increased levels of LPO, MDA and reduced activities of CAT and SOD in Model group as compared with Sham group. The levels of LPO and MDA were significantly decreased by about $130 \%$ and $50 \%$, and the activities of CAT and SOD were significantly increased by about $130 \%$ and $50 \%$ in CVBD and NMDP groups, as compared with Model group (Figure $3 \mathrm{~A}$ ).

The expression of Nrf2, HO-1, GCLM, NQO1 and LC-3B was detected by western blotting. After Nrf2, HO-1, GCLM and NQ01 being damaged by oxidative stress, the expression levels of these proteins decreased significantly in Model group as compared with Sham group. Compared with Model group, the protein expression levels in NMDP group and CVBD group were significantly higher than those in Model group, with the highest expression levels found in Nrf2 and NQ01 proteins (Figure 3B).

The expression level of autophagy-related protein LC-3B in Model group was higher than that in Sham group. The expression level of LC-3B in NMDP group was slightly lower than that in Model group, while this level was increased significantly in CVBD group than in the Model group (Figure 3C).

Establishing of OGD/R Model of HT22 Cells and the Pharmacodynamics Evaluation of CVBD Braintargeted Liposomes in vitro

Real-Time Live-Cell Imaging System and CCK-8 assay were used to detect cell fusion and cell viability in each group. Results (as shown in Figure 4A-C) showed that cell fused most in NC group after being cultured for the same long time, followed by CVBD-H and CVBD-M groups, with the cells fused least in the model and CVBD-L groups. The results from CCK-8 assay were consistent with those of cell fusion. Compared with NC group, the cell viability of Model group was significantly decreased by about $22 \%$, and such viability of CVBD-H group was significantly increased by about $13 \%$ as compared with Model group. 
The levels of ROS in the cells were measured. Results (as shown in Figure 4D) that ROS level in the OGD/R-induced Model group was significantly increased by about $34 \%$ as compared with NC group, and that level in CVBD-H group was dose-dependent decreased by about $28 \%$ as compared with Model group.

Effect of CVBD Brain-targeted Liposomes on Autophagy of HT22 Cells Damaged by OGD/R

The number of autophagosomes (yellow bright spots) and autophagolysosomes (red bright spots) in HT22 cells was significantly increased in Model group as compared with NC group. Compared with Model group, the number of autophagosomes in CVBD-L and CVBD-H groups increased further, but the number of autophagolysosomes did not change significantly. The endoplasmic reticulum (ER) probe was used to find that most autophagosomes were co-located with ER (Figure 5A, B).

After that, the expression levels of autophagy-related proteins were detected. The results showed that the levels of LC3 and p62 in NC group were very low, and the levels of LC3 and p62 in Model group and CVBD group were significantly increased. Besides, the expression level of LC3 protein in CVBD group was higher than that in Model group, while the expression level of p62 protein was lower, which supported the findings by using confocal laser scanning microscopy. In addition, the same changes in the expression levels of Beclin 1 protein and LC3 protein were found (Figure 5C).

The Protective Effect of CVBD Brain-targeted Liposomes on HT22 Cells Damaged by OGD/R was Investigated using the $\mathrm{CQ}$

The results showed that there was no significant difference in cell viability between Model group and CQ group. Significantly higher cell viability in CVBD-H+CQ group was found than Model group, but lower than CVBD-H group (Figure 6A).

We also tested ROS levels. The results showed that there was no significant difference in ROS levels between CQ group and Model group, and the cell viability decreased significantly in CVBD-H + CQ group as compared with Model group (Figure 6B).

Then we examined the expression levels of Nrf2, HO-1, GCLM and NQO1 after being damaged by oxidative stress. The results showed that the expression levels of these proteins in Model group and CQ group were significantly lower than those in NC group, while these levels in CVBD-H and CVBD-H + CQ groups were significantly higher than those in Model group (Figure 6C).

We also examined the expression levels of autophagy-related proteins. The results showed that the expression levels of LC3 and Beclin 1 proteins in CQ and NC groups were lower. The expression levels of LC3 and Beclin 1 in CVBD-H + CQ group were significantly higher than that in CQ group, but lower than that in CVBD-H group. The change in the expression level of p62 was opposite to that of LC3 (Figure 6D).

\section{Discussion}


Ischemic cerebrovascular disease (ICD) is one of the most important life-threatening disease all over the world, and it is essential to restore the blood supply in ischemic area to attenuate the damage of central nervous system during the treatment [22-23]. However, many studies have demonstrated that damage will be aggravated if the restore of blood supply begins after a certain time, which leads to a conclusion that inhibiting CIRI is of vital importance during the treatment of ICD [24-25].

CVBD, an alkaloid monomer extracted from Buxux microphylla, are well proved it can improve myocardial and cerebral ischemia. For example, Huang Yangning tablet, a CVBD-rich medication, has been clinical used as a first-line treatment for ICD. But in fact, the poor solubility in water and bioavailability of CVBD somehow lower down the therapeutic efficacy. In regard of the above defects, liposome may be a potential ideal carrier of CVBD for its better bioavailability, fewer toxic side-effects and higher targetaccuracy [26]. Meanwhile, most of drugs cannot be transferred into cerebrum because of BBB. Taken the above physiological characteristic into consideration, we have successfully designed a nasaladministrated CVBD brain-targeted liposomes and finished its Study on Pharmacokinetics of Blood-brain synchronization in the pre-study.

The primary studies have demonstrated that pathogenic mechanism of CIRI involves inflammation, oxidative stress, and autophagy and so on [27-29]. Plenty of free radicals could break through the inner anti-oxidative system. Then, LPO, MAD could further damage the surrounding tissue, causing inflammation, autophagy and apoptosis [30-31]. In addition, CIRI could results in damage and death of neurons in ischemic area. Some studies shows that neuron autophagy could be activated during the stroke, and the increase of neuron autophagy might be a potential treating target of damaged neurons in ischemic area [32].

In this study, we performed a MCAO model which can make a certain part into an ischemic area and precisely control the reperfusion time. It has been regarded as a classic model for its high similarity with human patient and good repeatability [33]. We found out that CVBD brain-targeted liposomes could improve the weight loss and neurological behavior assessment, reduce the size of ischemic necrosis and the damage of brain tissue, increase the amount of Nissl body, as well as restore the blood supply of damaged blood vessels, showing the protective effects of CVBD brain-targeted liposomes for CIRI. In addition, it could lower LPO and MAD levels, increase CAT and SOD levels, up-regulated the expression of Nrf2, HO-1, GCLM and NQ01, which can further keep the body in a balance of oxidative stress and attenuate the damages of brain tissue. The up-regulated LC-3B protein could also activate autophagy. Thus, we speculated the CVBD brain-targeted liposomes might promote the recovery of nerve function through the above pathway.

Next, the neuronal oxygen glucose deprivation/reoxygenation (OGD/R) model and HT22 cells were used to simulate the CIRI-induced neuronal injury to investigate the mechanism of CVBD brain-targeted liposomes in treating CIRI.

In the OGD/R, cerebral ischemia in vivo was simulated by deprivation of glucose in a hypoxic environment, and then the cells were transferred to an incubator with normal oxygen concentration and 
given normal complete medium to simulate ischemia-reperfusion injury. Based on the OGD/R studied by other scholars and the laboratory conditions, the OGD $9 \mathrm{~h} / \mathrm{R} 1 \mathrm{~h}$ was determined as the final modeling condition with gas concentration of $37{ }^{\circ} \mathrm{C}, 90 \% \mathrm{~N}_{2}$ and $5 \% \mathrm{O}_{2}$ [34-35].

The cell proliferation ability can be seen from the cell viability determined by CCK- 8 assay. Real-Time Live-Cell Imaging System was used to automatically take pictures at regular intervals to capture changes in the shape and area of cells in each field of vision. The effects of the compounds on cell proliferation/inhibition or survival/death were found by the values and curve of cell fusion (Phase Object Confluence (Percent)) [36]. Then the cell pharmacodynamics experiment was done to found that CVBD brain-targeted liposomes could improve the damage to cell fusion and cell viability caused by the OGD/R model in a dose-dependent manner. The CVBD-H group could increase $50 \%$ of the difference in values of cell viability between the control group and the model group. In this experiment, reactive oxygen species (ROS) level was also measured. ROS level is directly related to the damage caused by CIRI [37]. So the damage from oxidative stress by reducing ROS level is also one of the evaluation indicators of in vitro pharmacodynamics. It was found that the level of ROS in the CVBD-H group was statistically significantly lower than that in the model group.

Finally, mRFP-GFP-LC3 adenovirus, western blotting (WB) and chloroquine (CQ) were used to detect the autophagy and autophagic flux to investigate the mechanism of CVBD brain-targeted liposomes in the treatment of CIRI.

Autophagy is a dynamic process, including autophagic precursors, the formation of autophagosomes, the fusion of autolysosomes and degradation. The autophagic flux is typically used to show the whole dynamic process of autophagy [32,37]. The increased expression of autophagy genes may be due to the increase of autophagosomes or the decrease of autolysosome degradation. Meanwhile LC3 and p62 are important markers and substrates of autophagy and can reflect the activity of autophagosomes and the level of autophagic flux. The level of p62 protein was inversely proportional to the level of autophagic flux. Beclin 1 plays an essential role in the formation of autophagosomes. The results showed that CVBD brain-targeted liposomes could up-regulate the expression of LC3A/B- $\$ and Beclin 1, down-regulate the expression of p62, increase the number of yellow spots (autophagosomes), but not change the number of red spots (autolysosomes), as compared with Model group. These results suggest that CVBD braintargeted liposomes can activate autophagy of HT22 cells and increase the level of autophagic flux.

CQ, a common inhibitor of autophagy, was used to inhibit HT22 cells activated by OGD/R, and then CCK8 assay was performed and the level of ROS was measured. Besides, related proteins were determined by WB. The results showed that CQ could inhibit the cell fusion and cell viability in the OGD/R model, and increase ROS level. CQ + CVBD-H group could partly improve cell fusion and cell viability and lower ROS level, but the efficacy was not as good as using CVBD alone. CQ inhibited the expression of Nrf2, HO-1, GCLM and NQ01 damaged by antioxidant stress and autophagy-related proteins. CQ and high-dose CVBD could partially restore the inhibited protein expression, but not as effective as CVBD alone. 


\section{Conclusion}

In this study, we demonstrated that CVBD brain-targeted liposomes could effectively treat the cerebral ischemia-reperfusion injury by pharmacodynamics in vitro and vivo and studies on the related mechanism which involves reducing injury by oxidative stress and activating autophagy.

\section{Abbreviations}

CIRI, Cerebral ischemia-reperfusion injury; CVBD, Cyclovirobuxine D; MCAO, middle cerebral artery occlusion; TTC, triphenyl tetrazolium chloride; HE, hematoxylin-eosin; OGD/R, oxygen and glucose deprivation/re-oxygenation; LC3, Autophagy microvascular associated protein light chain 3; p62, Sequestosome 1; ROS, Reactive oxygen species; BBB, Blood brain barrier; EE, entrapment efficiency; PI, polydispersity index; NMDP, Nimodipine; DMEM, Dulbecco's modified Eagle's medium; FBS, fetal bovine serum; CCA, common carotid artery; ICA, internal carotid artery; ECA, external carotid artery; MCA, middle cerebral artery; LPO, Lipid peroxide; MDA, Malondialdehyde; CAT, Catalase; Nrf2, Nuclear factor erythroid 2related factor 2; HO-1, Heme oxygenase-1; NQ01, NADPH: quinone oxidoreductase 1; GCLM, Glutamatecysteine ligase regulatory subunit; GADPH, Glyceraldehyde-3-phosphate dehydrogenase; NC, Normal group; $C Q$, chloroquine; ICD, Ischemic cerebrovascular disease; SOD, Superoxide dismutase.

\section{Declarations}

\section{Acknowledgements}

We thank the reviewers for their valuable comments on this article.

\section{Funding}

This study was supported financially by Science and Technology Planning Project of Guangdong Province (Grants no. 20170211).

\section{Availability of data and materials}

Not applicable.

\section{Author contributions}

Tuo Liu, Fang Yang, Xiangyi Lu and Chang Liu conducted the research and analyzed data. Tuo Liu and Fang Yang mainly participated in data acquisition, analysis and wrote the manuscript drafting. Xiangyi Lu and Chang Liu participated in animal research. Tuo Liu revised the manuscript. Yang 
Yu, Zhilian Chen and Yongling Long supervised the study and revised the manuscript. All authors read and approved the fnal manuscript.

\section{Ethics statement}

The animal study was reviewed and approved by Animal Ethics Committee of Guangzhou University of Chinese Medicine, Guangzhou, China.

\section{Competing interests}

The authors declared that the research was conducted in the absence of any commercial or financial relationships that could be construed as a potential conflict of interest.

\section{Author details}

${ }^{1}$ School of Pharmaceutical Sciences, Guangzhou University of Chinese Medicine, Guangzhou, Guangdong, 510006, China. ${ }^{2}$ Institute of Chinese Materia Medica, China Academy of Chinese Medical Sciences, Beijing, 100070, China. ${ }^{3}$ Artemisinin Research Center, Institute of Chinese Materia Medica, China Academy of Chinese Medical Sciences, Beijing, 100070, China. ${ }^{4}$ The First Affiliated Hospital, Guangzhou University of Chinese Medicine, Guangzhou 510006, Guangdong, China.

\section{References}

1. Powers WJ, Rabinstein AA, Ackerson T, Adeoye OM, Bambakidis NC, Becker K, Biller J, Brown M, Demaerschalk BM, Hoh B, Jauch EC, Kidwell CS, Leslie-Mazwi TM, Ovbiagele B, Scott PA, Sheth KN, Southerland AM, Summers DV, Tirschwell DL,American Heart Association Stroke C (2018) 2018 Guidelines for the Early Management of Patients With Acute Ischemic Stroke: A Guideline for Healthcare Professionals From the American Heart Association/American Stroke Association. Stroke 49 (3): e46-e110

2. Wang $W$, Jiang $B$, Sun $H$, Ru X, Sun D, Wang L, Wang L, Jiang Y, Li Y, Wang Y, Chen Z, Wu S, Zhang Y, Wang D, Wang Y, Feigin VL,Investigators NE-C (2017) Prevalence, Incidence, and Mortality of Stroke in China: Results from a Nationwide Population-Based Survey of 480687 Adults. Circulation 135 (8): 759-771

3. Wu S, Cheng Y, Wu B,Liu M (2020) Stroke research in 2019: towards optimising treatment and prevention. The Lancet Neurology 19 (1): 2-3

4. Guo Q, Guo J, Yang R, Peng H, Zhao J, Li L,Peng S (2015) Cyclovirobuxine D Attenuates DoxorubicinInduced Cardiomyopathy by Suppression of Oxidative Damage and Mitochondrial Biogenesis Impairment. Oxid Med Cell Longev 2015: 151972 
5. Yan YY, Ao LY, Zhou L, Li CY, Fang WR, Shen WY, Liang BW, Zhu X,Li YM (2018) Therapeutic effects of JLX001 on cerebral ischemia through inhibiting platelet activation and thrombus formation in rats. Biomed Pharmacother 106: 805-812

6. Zhou L, Ao LY, Yan YY, Li WT, Ye AQ, Li CY, Shen WY, Liang BW, Xiong Z,Li YM (2019) JLX001 Ameliorates Ischemia/Reperfusion Injury by Reducing Neuronal Apoptosis via Down-Regulating JNK Signaling Pathway. Neuroscience 418: 189-204

7. Zhao B, Zhu J, Fei Y, Yin Q, Shen W, Liang B, Zhu X,Li Y (2020) JLX001 attenuates blood-brain barrier dysfunction in MCAO/R rats via activating the Wnt/beta-catenin signaling pathway. Life Sci 260: 118221

8. Li R, Huang Y, Chen L, Zhou H, Zhang M, Chang L, Shen H, Zhou M, Su P,Zhu D (2019) Targeted delivery of intranasally administered nanoparticles-mediated neuroprotective peptide NR2B9c to brain and neuron for treatment of ischemic stroke. Nanomedicine 18: 380-390

9. Migliore MM, Ortiz R, Dye S, Campbell RB, Amiji MM,Waszczak BL (2014) Neurotrophic and neuroprotective efficacy of intranasal GDNF in a rat model of Parkinson's disease. Neuroscience 274: $11-23$

10. Scafidi J, Hammond TR, Scafidi S, Ritter J, Jablonska B, Roncal M, Szigeti-Buck K, Coman D, Huang Y, McCarter RJ, Jr., Hyder F, Horvath TL,Gallo V (2014) Intranasal epidermal growth factor treatment rescues neonatal brain injury. Nature 506 (7487): 230-234

11. Vieira DB,Gamarra LF (2016) Getting into the brain: liposome-based strategies for effective drug delivery across the blood-brain barrier. Int J Nanomedicine 11: 5381-5414

12. Wang Y, Costanza F, Li C, Nimmagadda A, Song D,Cai J (2016) PEG-Poly(amino acid)s/MicroRNA Complex Nanoparticles Effectively Arrest the Growth and Metastasis of Colorectal Cancer. J Biomed Nanotechnol 12 (7): 1510-1519

13. Jiang L, He B, Pan D, Luo K, Yi Q,Gu Z (2016) Anti-Cancer Efficacy of Paclitaxel Loaded in pH Triggered Liposomes. J Biomed Nanotechnol 12 (1): 79-90

14. Song XL, Liu S, Jiang Y, Gu LY, Xiao Y, Wang X, Cheng L,Li XT (2017 Targeting vincristine plus tetrandrine liposomes modified with DSPE-PEG2000-transferrin in treatment of brain glioma. Eur $\mathrm{J}$ Pharm Sci 96: 129-140

15. Israel LL, Braubach O, Galstyan A, Chiechi A, Shatalova ES, Grodzinski Z, Ding H, Black KL, Ljubimova JY,Holler E (2019) A Combination of Tri-Leucine and Angiopep-2 Drives a Polyanionic Polymalic Acid Nanodrug Platform Across the Blood-Brain Barrier. ACS Nano 13 (2): 1253-1271

16. Zhu J, Zhang Y, Chen X, Zhang Y, Zhang K, Zheng H, Wei Y, Zheng H, Zhu J, Wu F, Piao JG, Zhu Z,Li F (2021) Angiopep-2 modified lipid-coated mesoporous silica nanoparticles for glioma targeting therapy overcoming BBB. Biochem Biophys Res Commun 534: 902-907

17. Wei H, Liu T, Jiang N, Zhou K, Yang K, Ning W,Yu Y (2018) A Novel Delivery System of Cyclovirobuxine D for Brain Targeting: Angiopep-Conjugated Polysorbate 80-Coated Liposomes via Intranasal Administration. J Biomed Nanotechnol 14 (7): 1252-1262 
18. Wei H, Lai S, Wei J, Yang L, Jiang N, Wang Q,Yu Y (2018) A Novel Delivery Method of Cyclovirobuxine D for Brain-Targeting: Chitosan Coated Nanoparticles Loading Cyclovirobuxine D by Intranasal Administration. J Nanosci Nanotechnol 18 (8): 5274-5282

19. Wei JB, Lai Q, Shumyak SP, Xu LF, Zhang CX, Ling JJ,Yu Y (2015) An LC/MS quantitative and microdialysis method for cyclovirobuxine $D$ pharmacokinetics in rat plasma and brain: The pharmacokinetic comparison of three different drug delivery routes. J Chromatogr B Analyt Technol Biomed Life Sci 1002: 185-193

20. Dergunova LV, Filippenkov IB, Stavchansky VV, Denisova AE, Yuzhakov VV, Mozerov SA, Gubsky LV,Limborska SA (2018) Genome-wide transcriptome analysis using RNA-Seq reveals a large number of differentially expressed genes in a transient MCAO rat model. BMC Genomics 19 (1): 655

21. Yu T, Guo F, Yu Y, Sun T, Ma D, Han J, Qian Y, Kryczek I, Sun D, Nagarsheth N, Chen Y, Chen H, Hong J, Zou W,Fang JY (2017) Fusobacterium nucleatum Promotes Chemoresistance to Colorectal Cancer by Modulating Autophagy. Cell 170 (3): 548-563 e516

22. Li G, Morris-Blanco KC, Lopez MS, Yang T, Zhao H, Vemuganti R,Luo Y (2018) Impact of microRNAs on ischemic stroke: From pre- to post-disease. Prog Neurobiol 163-164: 59-78

23. Ginsberg MD (2016) Expanding the concept of neuroprotection for acute ischemic stroke: The pivotal roles of reperfusion and the collateral circulation. Prog Neurobiol 145-146: 46-77

24. Leng T, Shi Y, Xiong ZG,Sun D (201 Proton-sensitive cation channels and ion exchangers in ischemic brain injury: new therapeutic targets for stroke? Prog Neurobiol 115: 189-209

25. Etherton MR, Gadhia RR,Schwamm LH (2020) Thrombolysis beyond $4.5 \mathrm{~h}$ in Acute Ischemic Stroke. Curr Neurol Neurosci Rep 20 (8): 35

26. Lu X, Dong J, Zheng D, Li X, Ding D,Xu H (2020) Reperfusion combined with intraarterial administration of resveratrol-loaded nanoparticles improved cerebral ischemia-reperfusion injury in rats. Nanomedicine 28: 102208

27. Chamorro Á, Dirnagl U, Urra X,Planas AM (2016) Neuroprotection in acute stroke: targeting excitotoxicity, oxidative and nitrosative stress, and inflammation. The Lancet Neurology 15 (8): 869881

28. Hayakawa K, Esposito E, Wang X, Terasaki Y, Liu Y, Xing C, Ji X,Lo EH (2016) Transfer of mitochondria from astrocytes to neurons after stroke. Nature 535 (7613): 551-555

29. Ham PB, 3rd,Raju R (2017) Mitochondrial function in hypoxic ischemic injury and influence of aging. Prog Neurobiol 157: 92-116

30. Zhou HJ, Li H, Shi MQ, Mao XN, Liu DL, Chang YR, Gan YM, Kuang X,Du JR (2017) Protective Effect of Klotho against Ischemic Brain Injury Is Associated with Inhibition of RIG-I/NF-kappaB Signaling. Front Pharmacol 8: 950

31. Qian J, Zhao X, Wang W, Zhang S, Hong Z, Chen X, Zhao Z, Hao C, Wang C, Lu S, Zhao B,Wang Y (2018) Transcriptomic Study Reveals Recovery of Impaired Astrocytes Contribute to Neuroprotective Effects of Danhong Injection Against Cerebral Ischemia/Reperfusion-Induced Injury. Front Pharmacol 9: 250 
32. Wang P, Shao BZ, Deng Z, Chen S, Yue Z,Miao CY (2018) Autophagy in ischemic stroke. Prog Neurobiol 163-164: 98-117

33. Liang J, Wang Q, Li JQ, Guo T,Yu D (2020) Long non-coding RNA MEG3 promotes cerebral ischemiareperfusion injury through increasing pyroptosis by targeting miR-485/AIM2 axis. Exp Neurol 325: 113139

34. Ran YC, Zhu M, Li SJ, Zhang ZX, Wang X, Zhang Y,Cheng JL (2018) Related Research and Recent Progress of Ischemic Penumbra. World Neurosurg 116: 5-13

35. Ryou MG,Mallet RT (2018) An In Vitro Oxygen-Glucose Deprivation Model for Studying IschemiaReperfusion Injury of Neuronal Cells. Methods Mol Biol 1717: 229-235

36. Lai S, Wei Y, Wu Q, Zhou K, Liu T, Zhang Y, Jiang N, Xiao W, Chen J, Liu Q,Yu Y (2019) Liposomes for effective drug delivery to the ocular posterior chamber. J Nanobiotechnology 17 (1): 64

37. Kaur J,Debnath J (2015) Autophagy at the crossroads of catabolism and anabolism. Nat Rev Mol Cell Biol 16 (8): 461-472

\section{Figures}



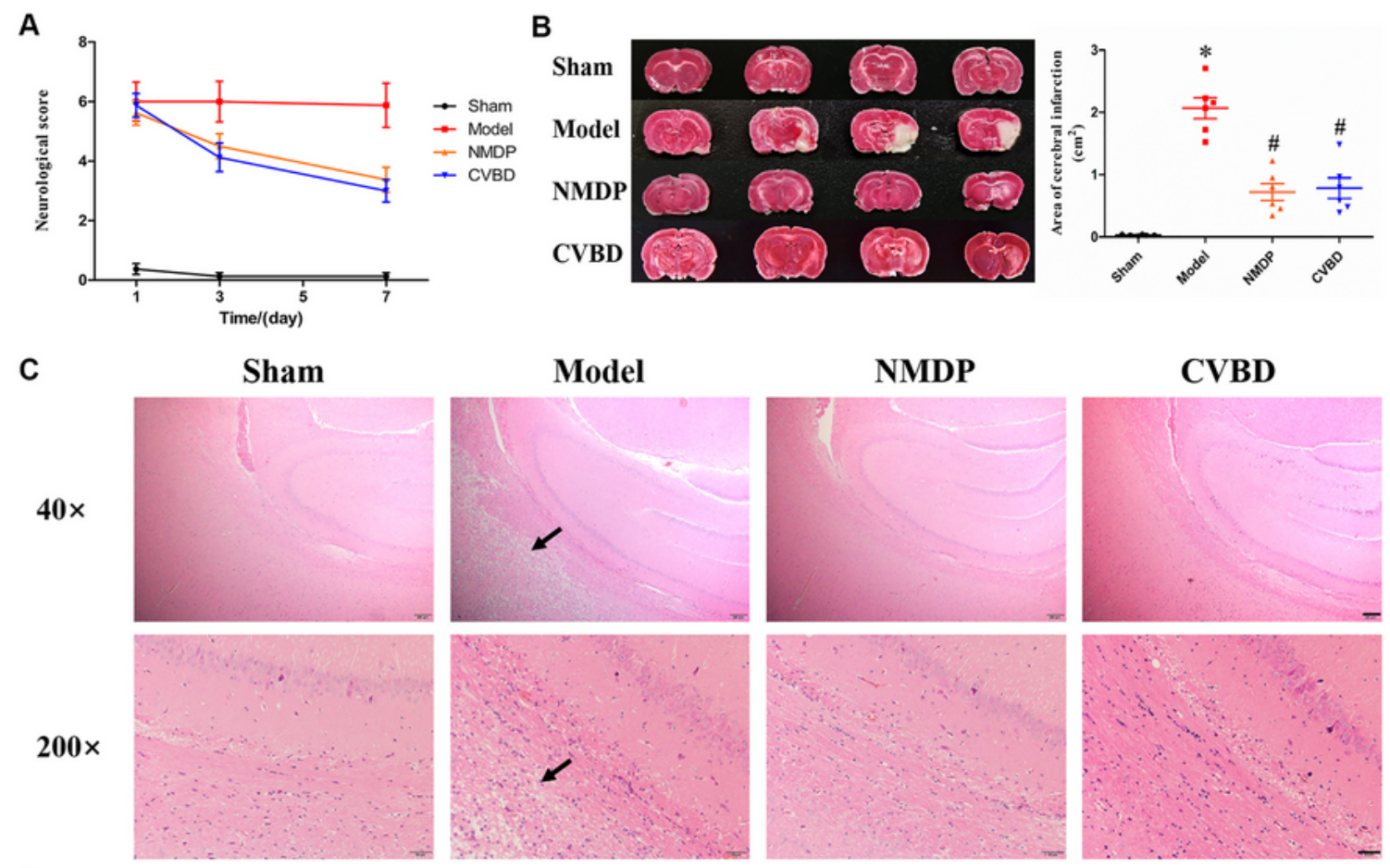

D
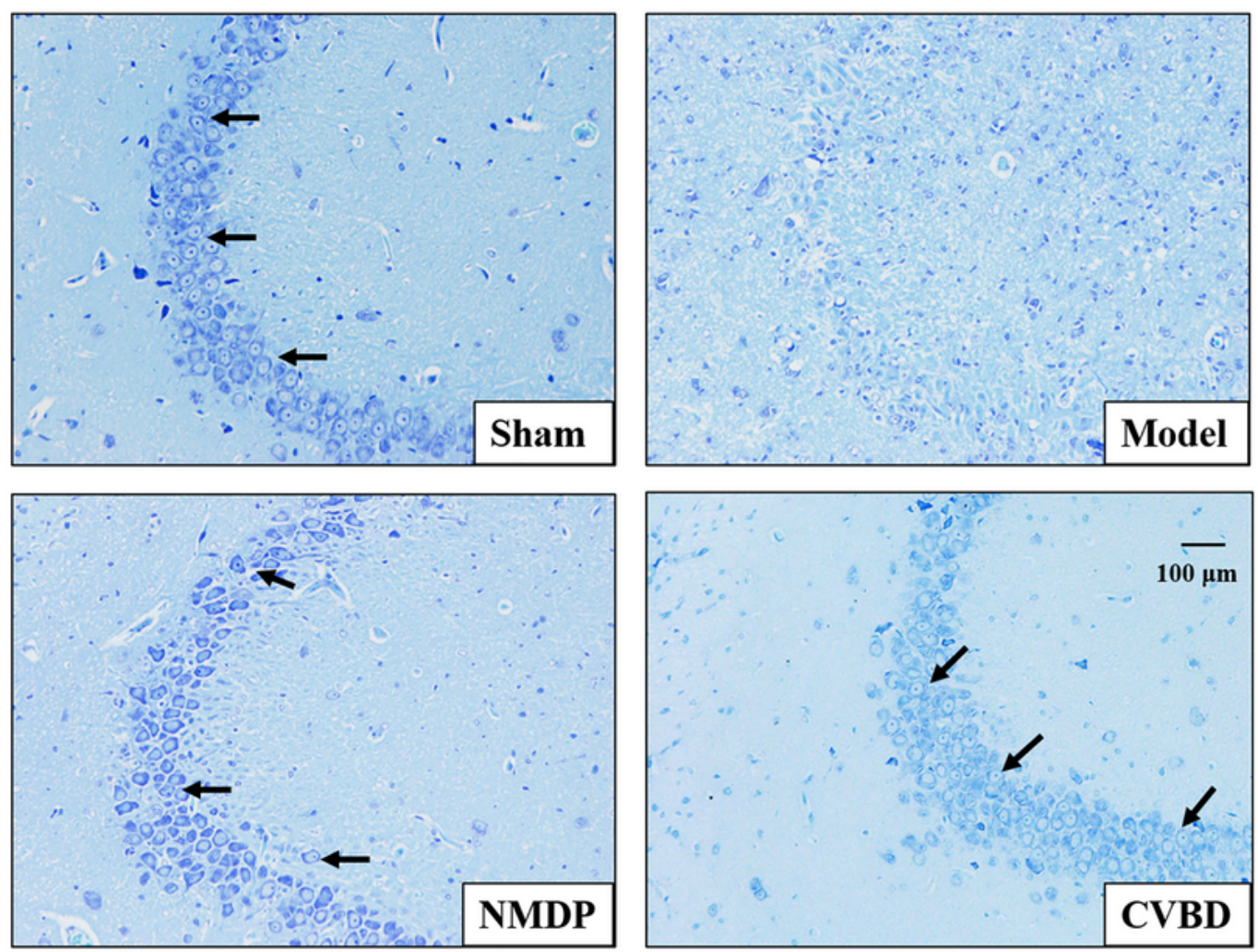

\section{Figure 1}

Neurological behavior assessment and cerebral infarction size. (A) Effect of CVBD brain-targeted liposomes on the neurological score of MCAO change in the groups. (B) Effect of CVBD brain-targeted liposomes on the area of cerebral infarction of MACO rats. (C) Effect of CVBD brain-targeted liposomes on the brain tissue of MACO rats, Black arrows point to pyramidal cells, HE staining, $\times 40, \times 200$. (D) Effect of CVBD brain-targeted liposomes on the brain tissue of MACO rats, Black arrows point to Nissl bodies, 
Nissl staining, $\times 200$. Data are expressed as the mean \pm SD. ${ }^{\star} P<0.05$ vs. Sham group; ${ }^{\#} P<0.05$ vs. Model group.

\section{Figure 2}

MRI Scanning. (A) Effect of CVBD brain-targeted liposomes on the cerebral morphology in MACO rats, MRI-T2 WI. (B) Effect of CVBD brain-targeted liposomes morphology of cerebrovascular in MACO rats, MRA-3D TOF.

\section{Figure 3}

The impact of CVBD brain-targeted liposomes on oxidative stress and autophagy. (A) Serum levels of LPO, MDA, and CAT and the activity of SOD in MACO rats after CVBD treatment. Data are expressed as the mean \pm SD. (B) Protein expression (Nrf2, HO-1, GCLM, and NQ01) in MACO rats after CVBD treatment. (C) LC3B Protein expression in MACO rats after CVBD treatment. Data are expressed as the mean \pm SD ( $n$ = 3). ${ }^{*} P<0.05$ vs. Sham group; ${ }^{\#} P<0.05$ vs. Model group. 

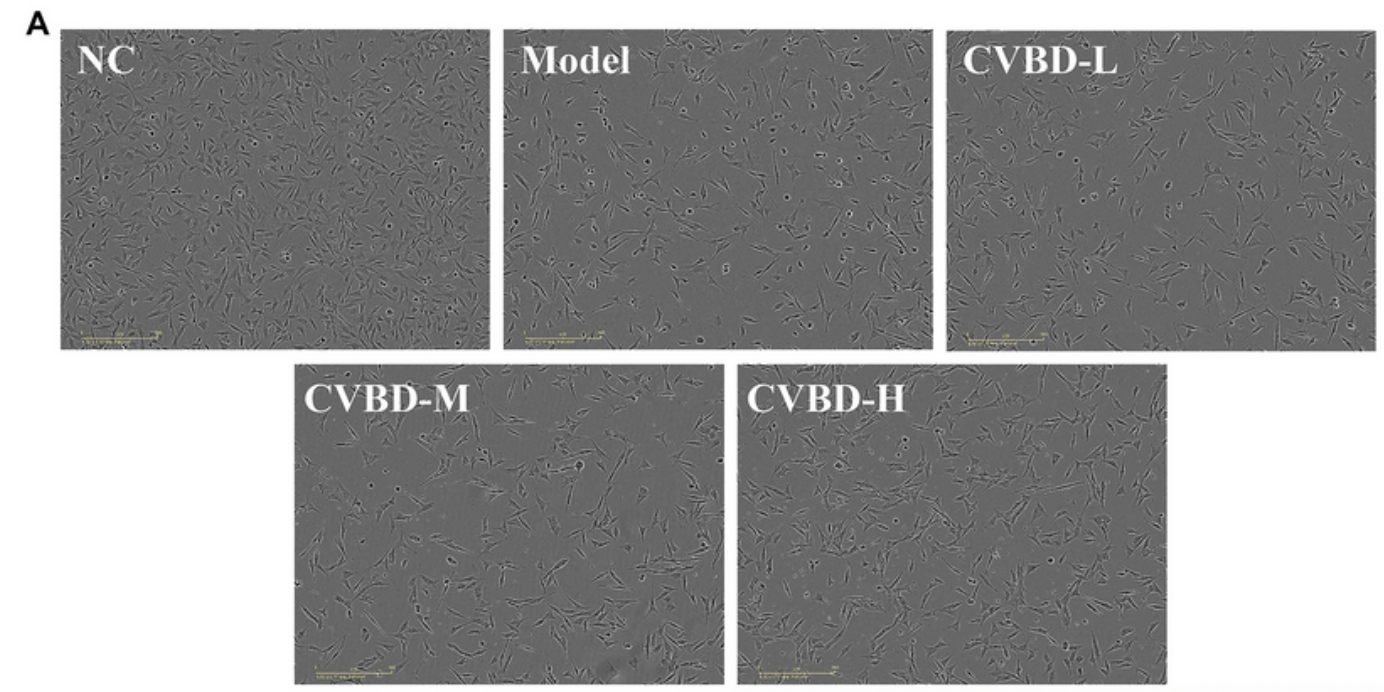

B

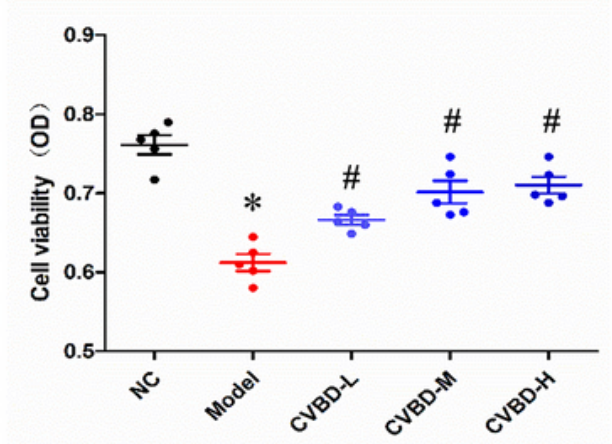

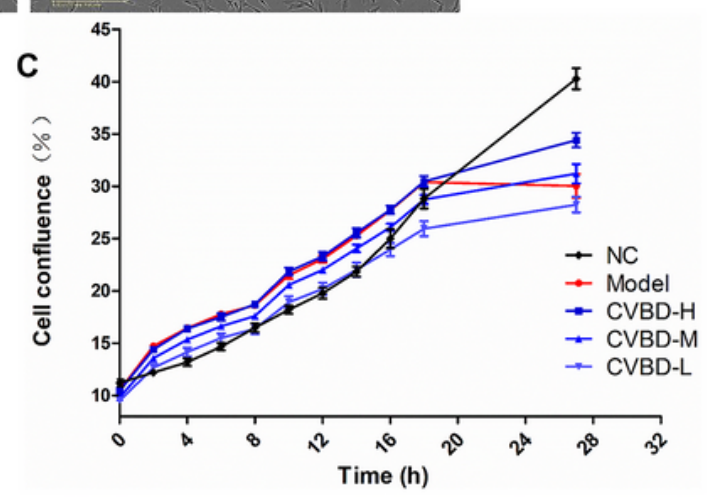
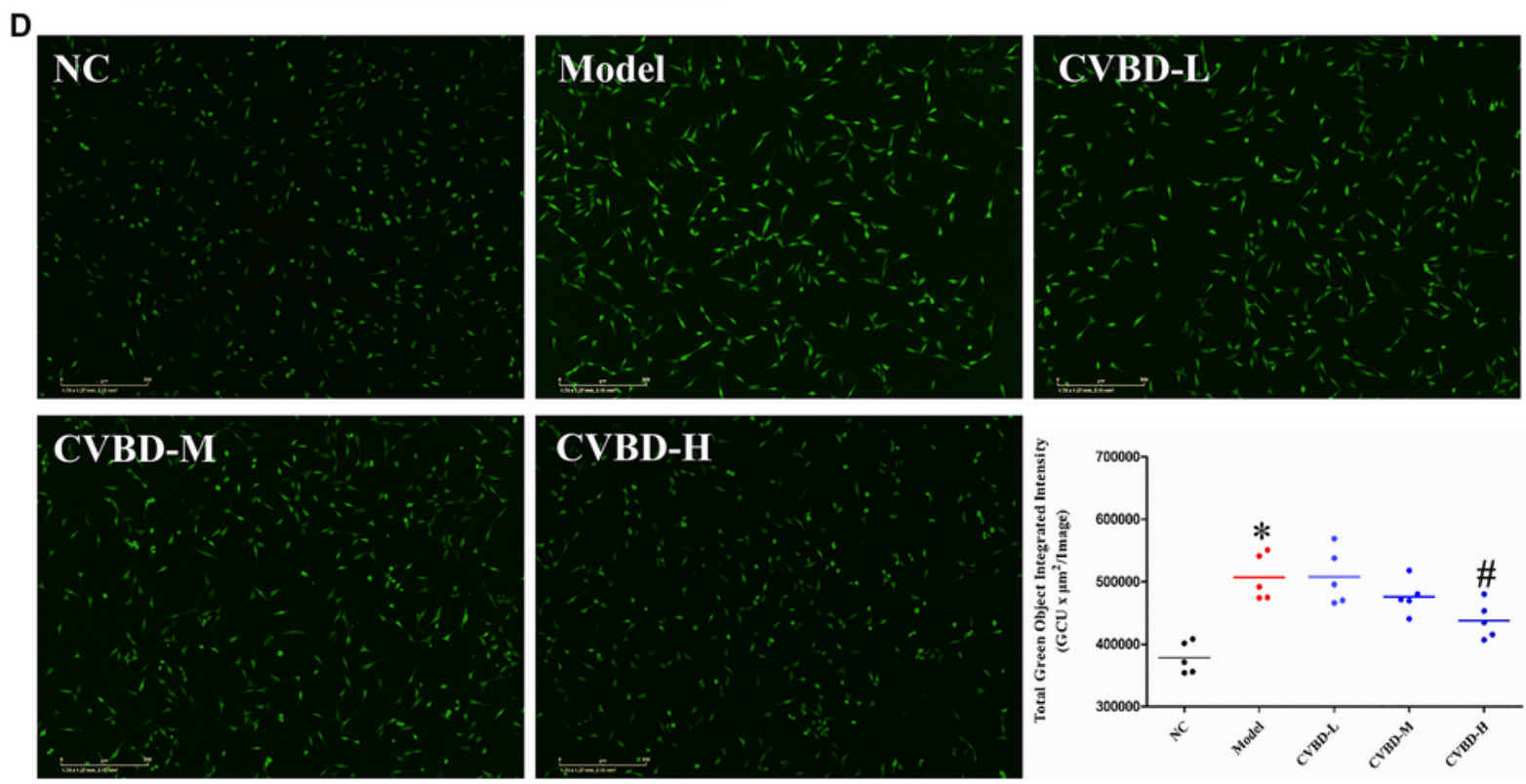

\section{Figure 4}

Pharmacodynamics evaluation in vitro and ROS level. (A) Cell confluence was determined by Real-Time Live-Cell Imaging System (×100). (B) Protective effect of CVBD brain-targeted liposomes in OGD/Rinjured HT22 cells by CCK8. Data were expressed as mean \pm SD, $n=5$. ${ }^{*} P<0.05$ vs. NC group; ${ }^{\#} P<0.05$ vs. Model group. (C) Cell fusion curve in $28 \mathrm{~h}$ by Real-Time Live-Cell Imaging System. Data were expressed 
as mean $\pm S D, n=5$. (D) Effect of CVBD brain-targeted liposomes on ROS level in OGD/R-injured HT22 cells $(\times 100)$. Data were expressed as mean $\pm \mathrm{SD}, n=5$. ${ }^{*} P<0.05$ vs. NC group; ${ }^{\#} P<0.05$ vs. Model group.

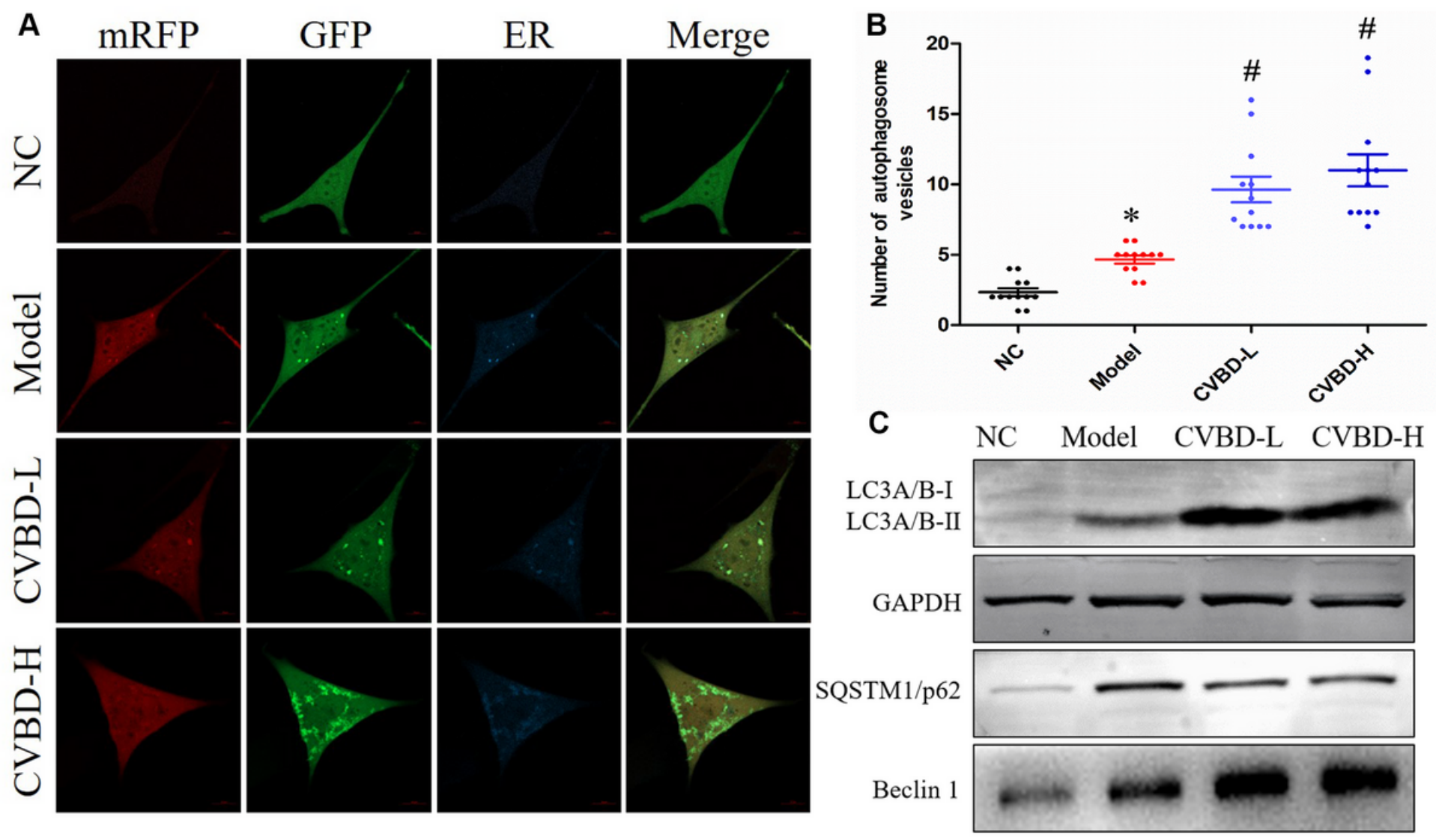

\section{Figure 5}

Cell autophagy. (A) Effect of CVBD brain-targeted liposomes on autophagy in OGD/R-injured HT22 cells. ( $\times 400)$. (B) Number of autophagosome vesicles. Data were expressed as mean $\pm S D, n=12 .{ }^{*} P<0.05 v s$. NC group; ${ }^{\#} P<0.05$ vs. Model group. (C) Protein expressions (LC3, Beclin 1, p62) in HT22 cells after CVBD brain-targeted liposomes treatment. Data were expressed as the mean $\pm \mathrm{SD}(n=3)$. ${ }^{*}<0.05 \mathrm{vs}$. NC group; ${ }^{\#} P<0.05$ vs. Model group.

\section{Figure 6}

The Protective Effect of CVBD Brain-targeted Liposomes on HT22 Cells Damaged by OGD/R. (A) Protective effect of CVBD-H or/and CQ in OGD/R-injured HT22 cells by CCK8. Data were expressed as mean \pm SD, $n=4$. ${ }^{*} P<0.05$ vs. NC group; ${ }^{*} P<0.05$ vs. Model group. (B) Effect of CVBD-H or/and CQ on ROS level in OGD/R-injured HT22 cells. Data were expressed as mean $\pm \mathrm{SD}, n=4 .{ }^{*} P<0.05$ vs. NC group; ${ }^{\#} P<0.05 \mathrm{vs}$. Model group. (C) Protein expressions of antioxidative stress. (D) Protein expressions of autophagy. 


\section{Supplementary Files}

This is a list of supplementary files associated with this preprint. Click to download.

- figureGraphicalAbstract.tif

- Sham3D.mov

- Model3D.mov

- CVBD3D.mov

- NMDP3D.mov 